\title{
Single-Speed Molecular Dynamics of Hard Parallel Squares and Cubes
}

\author{
W.G. Hoover • Carol G. Hoover • Marcus N. Bannerman
}

Received: 3 May 2009 / Accepted: 23 July 2009 / Published online: 30 July 2009

(C) The Author(s) 2009. This article is published with open access at Springerlink.com

\begin{abstract}
The fluid and solid equations of state for hard parallel squares and cubes are reinvestigated here over a wide range of densities. We use a novel single-speed version of molecular dynamics. Our results are compared with those from earlier simulations, as well as with the predictions of the virial series, the cell model, and Kirkwood's many-body singleoccupancy model. The single-occupancy model is applied to give the absolute entropy of the solid phases just as was done earlier for hard disks and hard spheres. As we should expect, the excellent agreement found here with all relevant previous work shows very clearly that configurational properties, such as the equation of state, do not require the maximumentropy Maxwell-Boltzmann velocity distribution. For both hard squares and hard cubes the free-volume theory provides a good description of the high-density solid-phase pressure. Hard parallel squares appear to exhibit a second-order melting transition at a density of 0.79 relative to close-packing. Hard parallel cubes have a more complicated equation of state, with several relatively-gentle curvature changes, but nothing so abrupt as to indicate a firstorder melting transition. Because the number-dependence for the cubes is relatively large the exact nature of the cube transition remains unknown.
\end{abstract}

Keywords Molecular dynamics $\cdot$ Computational methods $\cdot$ Melting transition

\section{Introduction}

Hard parallel squares and cubes have undergone extensive study [1-9]. Most of the hardparticle work motivating our present efforts is roughly 50 years old: Monte Carlo simu-

W.G. Hoover $(\bowtie) \cdot$ C.G. Hoover

Ruby Valley Research Institute, Highway Contract 60, Box 598, Ruby Valley 89833, NV, USA

e-mail: hooverwilliam@yahoo.com

M.N. Bannerman

School of Chemical Engineering and Analytical Science, The University of Manchester, Manchester M60 IQD, UK 
Fig. 1 A sample periodic configuration of $N=400$ hard parallel squares at two thirds the close-packed density, $V=600$. The figure illustrates a fluid. In the initial condition the squares with dots occupied the even-numbered rows of a perfect square lattice

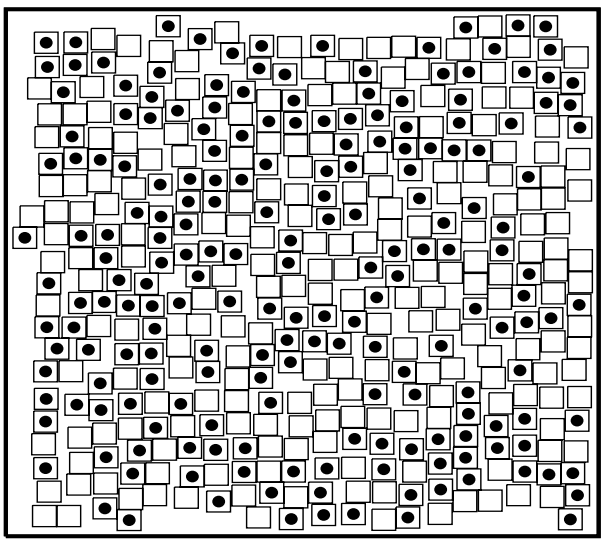

lation [6] indicated the absence of a first-order transition for hard parallel squares, while corresponding molecular dynamics simulations suggested its presence [5]. Because computers are now so much faster it is appropriate to reinvestigate this problem as well as the three-dimensional hard-cube analog.

In addition to the equilibrium equation of state, mixtures, transport coefficients, and various correlations have all been previously studied for squares and cubes. The most basic questions for statistical mechanics are the existence and nature of the melting transition for these two simple models. This question has been thoroughly settled for hard spheres, which exhibit a first-order transition between two coexisting phases, fluid and solid [10]. Despite hundreds of investigations, following the pioneering work of Alder, Jacobsen, Wainwright, and Wood [11, 12], the evidence is still not complete for disks, squares, and cubes [13, 14]. This uncertainty helped motivate the present work.

The two-dimensional squares model and its three-dimensional analog, the hard parallel cube model, are somewhat more tractable than disks and spheres because the square and cube potential functions are products of one-dimensional functions,

$$
\begin{array}{lrl}
\phi_{\text {squares }}=\phi(|x|) \phi(|y|) ; & \phi_{\text {cubes }}=\phi(|x|) \phi(|y|) \phi(|z|) ; \\
\phi(0<x<1)=\infty ; & \phi(x>1)=0 .
\end{array}
$$

The analytical simplicity due to these factorizations is a major motivation for the study of these systems, with an understanding of the melting transition a key goal. A good deal of the prior work lies twenty years or more in the past, so that today's enhanced computer speeds can lead to more precise conclusions than could the earlier work.

Throughout this work we set the mass and distance scales by imagining hard particles of unit mass and sidelength. The particles cannot rotate, acting as if their moments of inertia were infinite. The particles remain forever parallel, with their edges lined up with the $x, y$, and $z$ axes. See Fig. 1 for a sample two-dimensional fluid configuration.

The parallel square and cube models simplify the evaluation of the phase integrals derived from Gibbs' statistical mechanics. Both squares and cubes have fluid and solid phases, though until now the number-dependence of the dynamics and the thermodynamics has concealed the exact nature of the fluid-solid transitions. Gibbs' statistical mechanics shows that 
the pressure can be calculated from the "configurational integral" $Q_{N}(V, T)[15,16]$ :

$$
\begin{aligned}
& Q_{N} \equiv \int_{V} d r_{1} \cdots \int_{V} d r_{N} e^{-\Phi / k T} / N ! ; \quad \Phi=\sum_{i<j}^{N} \phi_{i j} ; \\
& P V / N k T=\left(\partial \ln Q_{N} / \partial \ln V\right)_{T} .
\end{aligned}
$$

$Q_{N}$ is the integral over all distinct arrangements of $N$ particles within a box of volume $V$ at the temperature $T . \Phi$ is the potential energy, either infinity or zero for the square and cube models. In the present work we set the energy scale by choosing Boltzmann's constant and the temperature equal to unity, $k T=1$.

The Mayers carried out an exact low-density series expansion of the pressure [15], the "virial expansion". For squares and cubes the series' coefficients, the virial coefficients, have been evaluated, analytically, through the seventh term [1-3]. A convenient extrapolation method for the series is provided by ratios of polynomials, "Padé approximants" [17, 18], of the type given in the Appendix.

At high density, where neither the density series nor its extrapolation are useful, a "freevolume" approach, exact near close packing $[19,20]$, can be used. For $D$-dimensional hard cubes of unit sidelength in a rigid box of sidelength $L=V^{1 / D}$, the configurational integral is $D N$-dimensional, but easy to approximate using ideas borrowed from Tonks' onedimensional work [21] and the Eyring-Hirschfelder cell model [22]. If for $D=3$ we assume that the cubes are ordered in $N^{2 / 3}$ columns parallel to the $z$ axis and allowed to move independently in the $x$ and $y$ directions, as in the self-consistent cell model of Fig. 2, the configurational integral over the $x$ and $y$ coordinates gives:

$$
\prod^{N}\left(\int d x \int d y\right) \rightarrow\left[(V / N)^{1 / 3}-1\right]^{2 N} .
$$

Because the arbitrary ordering of the particles can be chosen in $N$ ! distinct way, this ordering degeneracy exactly compensates for the factor of $1 / N$ ! in the definition of $Q$. The remaining integrals in the $z$ direction give Tonks' result for the one-dimensional hard-rod configurational integral:

$$
\begin{aligned}
\prod^{N}\left(\int d z\right) & \rightarrow\left[\left(V^{1 / 3}-N^{1 / 3}\right)^{N^{1 / 3}} /\left(N^{1 / 3}\right) !\right]^{N^{2 / 3}} \\
& \simeq\left[\left[(V / N)^{1 / 3}-1\right]^{N^{1 / 3}} e^{N^{1 / 3}}\right]^{N^{2 / 3}}=\left[(V / N)^{1 / 3}-1\right]^{N} e^{N},
\end{aligned}
$$

resulting in the lower bound:

$$
Q_{N}(V, T)>\left[(V / N)^{1 / 3}-1\right]^{3 N} e^{N} .
$$

For $D$-dimensional hard cubes the ordinary Eyring-Hirschfelder cell model exceeds this estimate by a factor of $\left(2^{D} / e\right)^{N}$. See the central illustration in Fig. 2 for a sketch of this cell model.

The free-volume equation of state results from either approach, the lower bound or the cell model,

$$
P V / N k T=1 /\left(1-\rho^{1 / D}\right) ; \quad \rho>(1 / 2)^{D} \text { for } D>1 .
$$




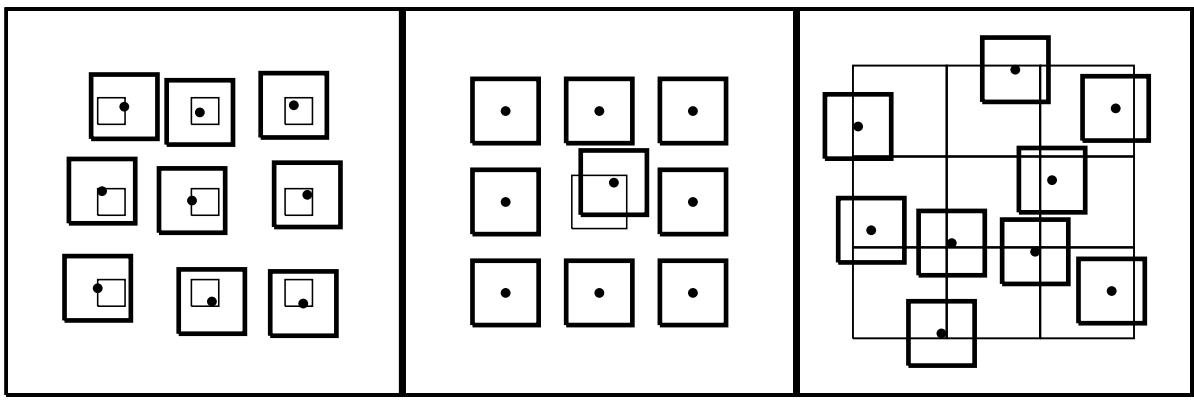

Fig. 2 The self-consistent cell model is shown at the left, and allows for the simultaneous independent motion of the centers (shown as dots) of all particles within the individual light squares of accessible states. In the self-consistent cell models all $N$ particles are treated alike. The more nearly accurate Eyring-Hirschfelder cell model shown in the center, has all the neighboring particles fixed while the central particle wanders over a much larger "free volume", four times bigger (for squares) than in the self-consistent case for $\rho>0.25$. The single-occupancy system, shown at the right, confines (the center of) each particle to a square of area $V / N$. Unlike the cell models, which reduce to simple one-body problems, the single-occupancy model is as complex to treat analytically as is the full unconstrained many-body problem

Our single-speed molecular dynamics results-see Sects. 4 and 5-suggest that this approximation is exact within terms of order unity, for hard parallel squares or cubes near close packing. For instance, a 128000 -collision simulation with 1000 hard parallel cubes at a density of 0.95 gave $P V / N k T=58.99 \pm 0.02$, equal to the free-volume compressibility factor, which is also 58.99 at this density. Our single-speed molecular dynamic results agree perfectly well with earlier results based on the Maxwell-Boltzmann velocity distribution.

This report is organized as follows. In Sect. 2 the Mayers' virial series is reviewed for squares and cubes. Section 3 describes the Eyring-Hirschfelder cell model approach to their thermodynamic properties. The cell model is specially useful for squares and cubes. We include here the details of Kirkwood's many-body single-occupancy model, a nearly exact description of the solid phase. Section 4 describes the kinetic theory used to analyze the molecular dynamics simulations. The simulations and their results are described in the following Sect. 5. Section 6 is devoted to the nature of the phase transition(s) for squares and cubes, with Sect. 7 a summary of our results and conclusions, including an attempt to reconcile our findings with the work of Jagla [13], Groh, and Mulder [14].

\section{Low Density and the Mayers' Virial Series}

There are plenty of theoretical approaches-series expansions, cell models, integral equations - to the equation of state and thermodynamic properties. Only one of them is rigorously correct - the Mayers' "virial expansion" of pressure as a series in the density [15]. This virial expansion gives a fairly good representation of the entire fluid equation of state for squares and for cubes. The hard-square and hard-cube virial series were carried out through seven terms in 1960 [2, 3]:

$$
\begin{aligned}
(P V / N k T)_{2 D}= & 1+2 \rho+3 \rho^{2}+3.66667 \rho^{3}+3.72222 \rho^{4}+3.02500 \rho^{5} \\
& +1.65065 \rho^{6}+\cdots,
\end{aligned}
$$




$$
\begin{aligned}
(P V / N k T)_{3 D}= & 1+4 \rho+9 \rho^{2}+11.33333 \rho^{3}+3.15972 \rho^{4}-18.87963 \rho^{5} \\
& -43.50543 \rho^{6}+\cdots .
\end{aligned}
$$

See again the Appendix for convenient Padé extrapolations of these truncated series. The negative $B_{6}$ and $B_{7}$ for cubes are notable as the first known instance in which hard particles definitely display negative (tensile) contributions to the virial expansion of the pressure. It is still unknown whether or not hard disks and hard spheres have such negative contributions.

In 1960 progress beyond $B_{7}$ was stalled by limited computer resources. The evaluation of $B_{7}$ required computing 468 separate integrals over the relative coordinates describing seven particles. The integrands are products of from seven to 21 of the Mayers' " $f$ functions",

$$
f(r)=e^{-\phi / k T}-1 .
$$

To simplify the integrals' evaluation Ree and Hoover introduced the identity

$$
1 \equiv e^{-\phi / k T}-f
$$

for all pairs of particles not linked by $f$ functions in the integrands, leading to a reduced number of integrals and to substantially better numerical accuracy in Monte Carlo calculations of the higher $B_{n}$. The number of integral types contributing to $B_{7}$ was reduced in this way from 468 to $171[17,18]$.

If, as is the case for hard disks, there were a melting transition for squares at about fourfifths the close-packed density, $\rho \simeq 0.80$, then the last of these known terms in the series, would make a contribution of about five percent to the total melting pressure. Techniques already developed for hard disks and spheres [18] could be applied to generate an additional three terms in the series. For $B_{8}, B_{9}$, and $B_{10} 2606,81564$, and 4980756 integral types need to be evaluated.

\section{High Density: The Eyring-Hirschfelder and Single Occupancy Models}

At higher density, near close packing, "cell models" are useful approximations. These models are based on the notion that particles sweep out a "free volume" bounded by their neighbors. Certain aspects of this idea are exactly correct $[19,20]$. This is the consequence of two facts: first, configurational properties are mass-independent in classical statistical mechanics; second, the dynamical evolution of a very light particle, moving rapidly in the presence of nearly stationary neighbors, does sweep out a free volume as time goes on. It should in fact be possible to derive the Mayers' virial series by considering this point of view in detail.

A much more complicated, but still cell-like, "single-occupancy" model can be constructed. This single-occupancy model gives a near-exact (within terms of order unity in $P V / N k T$ ) description of the solid phase. In the single-occupancy model each particle is constrained to one of $N$ nonoverlapping cells. Because vacancies and dislocations, as well as excursions outside such cells, are unimportant to the thermodynamics of the solid phase, the single-occupancy configurational integral,

$$
Q_{\mathrm{SO}} \equiv \int_{(V / N)} d r_{1} \cdots \int_{(V / N)} d r_{N} e^{-\Phi / k T}
$$

gives nearly the same solid-phase pressure-volume equation of state as does the exact configurational integral $Q_{N}$. Notice that the $1 / N$ ! appearing in $Q_{N}$ is absent in $Q_{\text {so }}$. This is 
because each particle is restricted to occupy a particular cell. By including collisions with cell walls it is easy to modify a molecular dynamics simulation to compute single-occupancy properties, as we detail in Sect. 5.

Besides exact free-volume measurements [20], there are several approximate methods for estimating the free volume. In the self-consistent cell model, all particles are distributed so near their lattice sites that no overlaps can occur. In the alternative inconsistent, but more nearly accurate, Eyring-Hirschfelder cell model, the motion of a single particle is considered, with all its neighbors held fixed at their lattice sites. In either case the approximate partition function includes the $N$ th power of the cell-model free volume:

$$
Z(N, V, T) \equiv v_{f}^{N} / \lambda^{D N} ; \quad \lambda^{2}=h^{2} / 2 \pi m k T .
$$

As is usual $h$ is Planck's constant and $\lambda$ is de Broglie's wavelength. Both of the cell models and the single-occupancy model are illustrated for hard parallel squares in Fig. 2.

At high density, these forms of the cell model, plus various approximate bounds on the hard square partition function all suggest that the "free volume" equation of state:

$$
P V / N k T=1 /\left(1-\rho^{1 / D}\right)
$$

is asymptotically correct near the close-packed limit, $\rho \rightarrow 1$. At a density of $2^{-D}$ with $D>1$ the Eyring-Hirschfelder cell model allows the central "wanderer" particle to escape its cell. The free volume changes there, discontinuously, from an intensive localized volume to a netlike extensive volume-the total volume $V$ less the exclusion volumes of the $N-1$ particles fixed at their lattice sites. At this "percolation transition" $[9,20]$ the model pressure jumps from the free-volume value, $\rho k T /\left(1-\rho^{1 / D}\right)$, to infinity.

Monte Carlo hard-square simulations showing the absence of a sharp fluid-solid transition [5] contradict molecular dynamics work [4, 6], also carried out in the early 1970s. The molecular dynamics results suggested a van der Waals loop joining the two phases. In the present work we measure the equation of state using molecular dynamics with the special single-speed velocity distribution described in the next section. We also use singleoccupancy simulation results to measure the solid-phase entropy directly.

\section{Single-Speed Molecular Dynamics for Squares and Cubes}

The factorization of the partition function into a kinetic part and a configurational part suggests that any reasonable velocity distribution, with vanishing total momentum and capable of reaching all configurations, can be used for computing configurational properties. In the present work we choose the $x$ and $y$ and $z$ velocity components all equal to \pm 1 , corresponding to unit isotropic temperature:

$$
v_{x}^{2}=v_{y}^{2}=v_{z}^{2} \equiv k T / m=1 .
$$

Parallel hard squares and cubes move and collide as if their moments of inertia were infinite. The particles do not rotate when they collide, but simply exchange $x$ or $y$ or $z$ momenta (in the center of mass system of coordinates) on collision. Thus the velocity distribution is unchanged by particle collisions. In single-occupancy simulations the cell walls change this. Then the center of each square or cube is confined to an individual cell of volume $V / N$. Collisions at the cell walls simply reflect the $x$ or $y$ or $z$ momentum perpendicular to the 
confining wall. Whenever a particle is reflected by a cell wall the center-of-mass momentum is shifted, by $\pm 2 / N$.

In all of our simulations the number of particles, the density, and the temperature are fixed. From the measured all-pairs particle-particle collision rate we determine the pressure. In the single-occupancy case note that the cell walls make no special nonideal contribution to the pressure. The kinetic part of the pressure is still given by $(P V / N k T)_{K}=1$. We choose to calculate the total pressure directly from the measured all-pairs collision rate $\Gamma$, using the exact relation:

$$
P V / N k T=(P V / N k T)_{K}+(P V / N k T)_{\Phi}=1+B_{2} \rho\left(\Gamma / \Gamma_{0}\right) .
$$

The dot product $(F \cdot r)_{i j}$ is the same for every collision:

$$
(F \cdot r)_{i j} \equiv F_{i j} \cdot r_{i j} \equiv-\nabla_{i} \phi_{i j} \cdot\left(r_{i}-r_{j}\right)=2 k T .
$$

The time average, which gives the potential contribution to $P V$, is computed by summing all the $C$ collisional $i j$ pair contributions taking place during the sufficiently long time $t$ :

$$
(1 / t) \sum_{C}(F \cdot r)_{i j}=(1 / t) \sum_{C} 2 k T .
$$

As a consequence, the "virial-theorem pressure" with single-speed dynamics is identical to the "collision-rate pressure":

$$
P V / N k T=1+(1 / D N k T) \sum_{i<j}\left\langle(F \cdot r)_{i j}\right\rangle=1+B_{2} \rho\left(\Gamma / \Gamma_{0}\right) .
$$

The low-density collision rate $\Gamma_{0}$ can be calculated in either of two different ways, both leading to the same result. A relatively complex approach is to calculate separate crosssections and collision probabilities for relative speeds of $( \pm \sqrt{4}, \pm \sqrt{8}, \pm \sqrt{12})$ (for cubes). The simpler approach multiplies the probability for a collision of cubes $i$ and $j$ in the $x$ direction by 3 and by $N(N-1) / 2$, the number of pairs of particles, giving:

$$
\begin{array}{ll}
\left(\Gamma_{0} / N\right)=2 \rho & \text { (squares) } \\
\left(\Gamma_{0} / N\right)=6 \rho & \text { (cubes) } .
\end{array}
$$

To confirm these simple relations and to check that the single-speed dynamics gives the same pressure as does Maxwell-Boltzmann dynamics, we measured the collision rate for 1000 cubes at a density of 0.1 for a run with 512000 collisions. The collision rate per particle (collisions per unit time divided by the total number of particles) was 0.751669 , giving a compressibility factor of

$$
P V / N k T=1+0.1 \times 4 \times(0.751669 / 0.6)=1.5011 \pm 0.0001,
$$

in excellent agreement with van Swol and Woodcock's 1987 calculation [9], 1.5016 \pm 0.004 . We must stress that the simple velocity distribution $( \pm 1, \pm 1, \pm 1)$, because the system is configurationally ergodic, gives the same pressure as would a Maxwell-Boltzmann distribution (though with considerably less effort).

Both the low-density and high-density regions are well understood for squares and cubes. Our main interest is in the square and cube analogs of what Wood aptly called "the region of 
confusion" for hard disks, where the fluid and solid phases come and go, but with a pace so slow that meaningful averages are hard to obtain. We emphasize the region of confusion in the following two Sections, which are devoted to the results of our simulations. Our singleoccupancy results, together with thermodynamic integration,

$$
d(S / N k)_{T}=-(P V / N k T) d \ln \rho,
$$

make it possible to determine the relative stabilities of the fluid and solid phases as functions of density.

\section{Pressure and Entropy from Single-Speed Molecular Dynamics}

\subsection{Pressure Data}

To make contact with earlier work, and to provide data for thermodynamic integration we have considered a wide range of densities for squares and cubes. Tables 1 and 2 compare a small sampling of the single-speed molecular dynamics data of the types shown in Figs. 3 and 4 . For both squares and for cubes, these data include both conventional and singleoccupancy predictions, as well as the pressure and entropy predictions of the truncated virial series, the Padé approximant, and the self-consistent cell model. Although we have carried out a wide range of simulations, with density spacings of 0.01 or 0.005 and a wide range of system sizes, we list here only two sets of data, sufficient that other workers could easily check the consistency of their calculations with ours. The tabulated data, as well as those shown in the figures, are quite representative of our body of results, and have been chosen so that the reader can see the relative usefulness of the various virial series and cell models to predicting and interpreting the dynamical data.

Table 1 Compressibility factor and reduced entropy (relative to an ideal gas at the same density and temperature) for 400 hard parallel squares with 40,000 collisions at each density. $s \equiv\left(S_{\rho}-S_{\text {ideal }}\right) / N k$

\begin{tabular}{lllllllllll}
\hline$\rho$ & $Z_{M D}$ & $Z_{\text {virial }}$ & $Z_{\text {Padé }}$ & $Z_{\mathrm{SO}}$ & $Z_{\mathrm{FV}}$ & $s_{\mathrm{MD}}$ & $s_{\text {virial }}$ & $s_{\text {Padé }}$ & $s_{\mathrm{SO}}$ & $s_{\mathrm{FV}}$ \\
\hline 0.05 & 1.108 & 1.108 & 1.108 & 1.032 & 1.288 & -0.105 & -0.104 & -0.104 & -1.022 & -1.506 \\
0.10 & 1.231 & 1.234 & 1.234 & 1.095 & 1.462 & -0.217 & -0.216 & -0.217 & -1.062 & -1.760 \\
0.15 & 1.382 & 1.382 & 1.382 & 1.184 & 1.632 & -0.339 & -0.338 & -0.339 & -1.116 & -1.980 \\
0.20 & 1.551 & 1.556 & 1.556 & 1.304 & 1.809 & -0.472 & -0.471 & -0.472 & -1.185 & -2.186 \\
0.25 & 1.760 & 1.763 & 1.763 & 1.459 & 2.000 & -0.618 & -0.617 & -0.617 & -1.269 & -2.386 \\
0.30 & 2.005 & 2.008 & 2.008 & 1.657 & 2.211 & -0.778 & -0.777 & -0.778 & -1.369 & -2.587 \\
0.35 & 2.317 & 2.299 & 2.299 & 1.897 & 2.449 & -0.955 & -0.954 & -0.954 & -1.488 & -2.791 \\
0.40 & 2.647 & 2.648 & 2.646 & 2.182 & 2.721 & -1.151 & -1.149 & -1.150 & -1.626 & -3.002 \\
0.45 & 3.058 & 3.064 & 3.059 & 2.524 & 3.038 & -1.369 & -1.367 & -1.367 & -1.784 & -3.222 \\
0.50 & 3.541 & 3.561 & 3.550 & 2.931 & 3.414 & -1.611 & -1.609 & -1.608 & -1.965 & -3.456 \\
0.55 & 4.096 & 4.156 & 4.135 & 3.419 & 3.870 & -1.882 & -1.880 & -1.878 & -2.171 & -3.707 \\
0.60 & 4.782 & 4.867 & 4.833 & 4.022 & 4.436 & -2.184 & -2.184 & -2.180 & -2.407 & -3.980 \\
0.65 & 5.586 & 5.714 & 5.678 & 4.805 & 5.161 & -2.523 & -2.526 & -2.519 & -2.678 & -4.282 \\
0.70 & 6.349 & 6.724 & 6.740 & 5.838 & 6.122 & -2.903 & -2.912 & -2.903 & -2.996 & -4.624 \\
0.75 & 7.575 & 7.924 & 8.181 & 7.268 & 7.464 & -3.329 & -3.346 & -3.345 & -3.376 & -5.020 \\
0.80 & 9.476 & 9.346 & 10.426 & 9.339 & 9.472 & -3.814 & -3.837 & -3.874 & -3.842 & -5.497 \\
\hline
\end{tabular}


Table 2 Compressibility factor and reduced entropy (relative to an ideal gas at the same density and temperature) for 1000 fluid hard parallel cubes and 1000 single-occupancy hard parallel cubes with 1,000,000 collisions at each density. $s \equiv\left(S_{\rho}-S_{\text {ideal }}\right) / N k$

\begin{tabular}{lllllllllll}
\hline$\rho$ & $Z_{M D}$ & $Z_{\text {virial }}$ & $Z_{\text {Padé }}$ & $Z_{\mathrm{SO}}$ & $Z_{\mathrm{FV}}$ & $s_{\mathrm{MD}}$ & $s_{\text {virial }}$ & $s_{\text {Padé }}$ & $s_{\mathrm{SO}}$ & $s_{\mathrm{FV}}$ \\
\hline 0.05 & 1.224 & 1.224 & 1.224 & 1.129 & 1.583 & -0.212 & -0.212 & -0.212 & -1.100 & -2.379 \\
0.10 & 1.501 & 1.501 & 1.501 & 1.346 & 1.866 & -0.449 & -0.449 & -0.449 & -1.252 & -2.872 \\
0.15 & 1.840 & 1.840 & 1.840 & 1.641 & 2.134 & -0.715 & -0.715 & -0.715 & -1.446 & -3.274 \\
0.20 & 2.246 & 2.247 & 2.247 & 1.995 & 2.408 & -1.011 & -1.011 & -1.011 & -1.678 & -3.637 \\
0.25 & 2.725 & 2.723 & 2.721 & 2.392 & 2.702 & -1.339 & -1.339 & -1.339 & -1.942 & -3.982 \\
0.30 & 3.261 & 3.264 & 3.260 & 2.804 & 3.025 & -1.700 & -1.700 & -1.699 & -2.232 & -4.321 \\
0.35 & 3.845 & 3.857 & 3.850 & 3.197 & 3.387 & -2.092 & -2.093 & -2.091 & -2.539 & -4.660 \\
0.40 & 4.464 & 4.475 & 4.472 & 3.597 & 3.799 & -2.512 & -2.515 & -2.512 & -2.858 & -5.005 \\
0.45 & 5.085 & 5.075 & 5.101 & 4.081 & 4.279 & -2.955 & -2.959 & -2.958 & -3.191 & -5.361 \\
0.50 & 5.528 & 5.594 & 5.713 & 4.676 & 4.847 & -3.412 & -3.416 & -3.422 & -3.545 & -5.735 \\
0.55 & 5.863 & 5.943 & 6.288 & 5.410 & 5.535 & -3.857 & -3.872 & -3.898 & -3.929 & -6.133 \\
0.60 & 6.648 & 6.000 & 6.815 & 6.317 & 6.387 & -4.309 & -4.307 & -4.381 & -4.351 & -6.563 \\
0.65 & 7.496 & 5.608 & 7.292 & 7.436 & 7.476 & -4.785 & -4.695 & -4.866 & -4.818 & -7.035 \\
0.70 & 8.920 & 4.565 & 7.725 & 8.904 & 8.921 & -5.315 & -5.003 & -5.348 & -5.346 & -7.565 \\
\hline
\end{tabular}

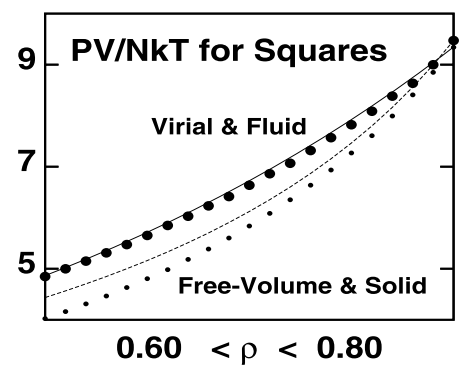

Fig. 3 Compressibility factor for 400 hard parallel squares (dots) compared with the predictions (lines) of the 7-term virial series and the free-volume theory. The upper set of larger dots represents unconstrained molecular dynamics while the lower set of smaller dots represents single-occupancy simulations. The fluid points correspond to 400000 collisions each; the solid points correspond to 4000000 collisions each. The dots shown represent 21 simulations, equally spaced in density from 0.60 to 0.80 , inclusive

The results we tabulate for squares (in the range $0.40 \leq \rho \leq 0.65$ ) in Table 1 show that some of the higher virial coefficients from the Padé approximant are negative (because adding in the higher contributions reduces the sum below that of the truncated series). In general, for cubes as well as squares, the truncated series are just as useful as are the Padé approximants. There is a significant difference between the two approaches, truncated and Padé, beginning, for squares, at a density of about 0.70 and, for cubes, at a density of about 0.50 . There are also enhanced fluctuations just beyond these densities, so that the pressure data by themselves leave the exact nature of the fluid-solid phase transition somewhat nebulous. Despite this uncertainty, the present data certainly show that the van der Waals loop found in the earlier dynamics work [7] was an artefact of the short computer runs which were possible in the early 1970 s. 


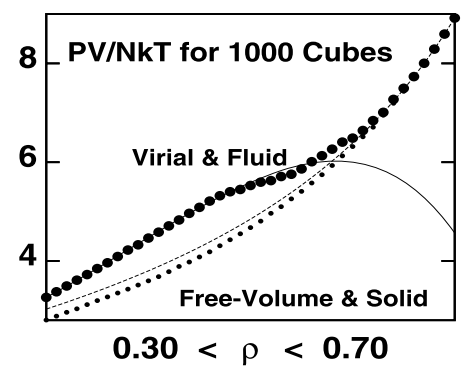

Fig. 4 Compressibility factor for 1000 hard parallel cubes (dots) compared with the predictions (lines) of the 7-term virial series and the free-volume theory. The upper set of dots represents unconstrained molecular dynamics while the lower set represents single-occupancy simulations. Each point corresponds to a simulation with a million collisions. The dots shown represent 41 simulations, equally spaced in density from 0.30 to 0.70 , inclusive

The results for cubes in Table 2, and plotted in Fig. 4 with many additional points, do lead to one relatively straightforward conclusion: for cubes there is no suggestion of a first-order phase transition. The jumpy nature of the cube equation of state for systems with less than 1000 particles disappears for longer runs and larger systems. Even a discontinuity in slope (second-order transition) looks doubtful for cubes.

In both two and three dimensions the free-volume equation of state is evidently exact, within terms of order unity, near close packing. At the same time it is hard to predict with great confidence precisely where the transition from fluid to solid is located or what its order might be from pressure data alone.

In an attempt better to locate and characterize the square and cube fluid-solid phase transitions we investigated the single-occupancy entropy approach described in the following subsection. This same approach was successful forty years ago in interpreting hard-disk and hard-sphere simulations [10].

\subsection{Entropy Calculations and the Solid-Phase Entropy Constant}

Two thermodynamic phases with the same pressure, temperature, composition, and Gibbs' free energy per particle,

$$
(G / N k T)=(E / N k T)+(P V / N k T)-(S / N k),
$$

are in equilibrium with one another. For squares and cubes the energies of the fluid and solid are purely kinetic, $k T / 2$ per degree of freedom, so that the only difficulty in comparing free energies lies in estimating the entropy $S$. Ree and Hoover $[10,16,17]$ showed how to implement Kirkwood's single-occupancy thermodynamics [23] so as to measure the entropy in the solid phase, $S_{\text {solid }} \simeq S_{\text {So. The cell-cluster theory is an alternative approach and was }}$ successful for hard squares $[6,24]$. So far as we know this theory has not been applied to parallel cubes until now.

The somewhat inconclusive nature of the pressure plots (Figs. 3 and 4) led us to consider separate calculations of the entropy for both phases, fluid and solid. Knowing the entropy is equivalent to knowing the free energy for hard particles. The fluid phase is no problem. From the virial series, the entropy, relative to that of an ideal gas at the same density and temperature, can be expressed in terms of the virial coefficients,

$$
(S / N k)-(S / N k)_{\text {ideal }}=-B_{2} \rho-\left(B_{3} \rho^{2} / 2\right)-\left(B_{4} \rho^{3} / 3\right)-\left(B_{5} \rho^{4} / 4\right)-\cdots .
$$


The fluid-phase entropies for squares and cubes appear in Tables 1 and 2. The analytic virial-series entropy, Padé approximant entropy, and the entropy from integrated molecular dynamics pressures are included there.

To calculate the isothermal solid-phase entropy we can use direct integration of the single-occupancy equation of state:

$$
d(S / N k)_{T}=-(P V / N k T) d \ln \rho .
$$

It is convenient to integrate the compressibility-factor difference,

$$
(\Delta S / N k)_{\rho}=\left[(S / N k)_{\mathrm{SO}}-(S / N k)_{\mathrm{FV}}\right]_{\rho}=\int_{0}^{\rho}\left[(P V / N k T)_{\mathrm{FV}}-(P V / N k T)_{\mathrm{SO}}\right] d \ln \rho^{\prime},
$$

using the known low-density values as the initial condition at $\rho=0.01$ :

$$
\begin{aligned}
{[\rho \simeq 0] } & \longrightarrow\left[S_{\mathrm{SO}}=S_{M D}-N k=S_{\mathrm{FV}}\right] \\
S_{\mathrm{SO}} & \left.\rightarrow\left\{-1-2 \rho^{3 / 2},-1-6 \rho^{4 / 3}\right\} \text { for } \text { \{squares, cubes }\right\} .
\end{aligned}
$$

These limiting cases result if the lowest-order term in a Mayer $f$-function expansion of the single-occupancy partition function is worked out [25]. Apart from the factor $-\rho / V$ this pair interaction term corresponds to the product of (i) the number of shared nearest-neighbor cell walls ( $2 N$ for squares and $3 N$ for cubes) and (ii) the two-particle integral in the vicinity of such a wall, $\left[2 \rho^{-1 / 2} / 2\right.$ for squares and $4 \rho^{-2 / 3} / 2$ for cubes]. Such a calculation was detailed for hard disks and spheres in 1967 [25]. Because the single-occupancy pressure data are smooth and regular, without large fluctuations, the numerical integrations are relatively easy to perform, for both squares and cubes. With a few dozen points the trapezoidal rule can easily achieve an accuracy of $\pm 0.01 N k$.

Straightforward numerical integration of the single-occupancy data, using the thermodynamic relation,

$$
\Delta S / N k=\int-P V / N k T d \ln \rho,
$$

shows that the entropy for hard squares, at densities of 0.82 and above, exceeds that of the Eyring-Hirschfelder cell model by $s_{0}$ (squares) $=0.27_{3} N k$, in precise agreement with the Rees' calculation [6] as well as the corresponding result for hard disks [17]. The last row of data in Table 1 give the estimate (at $\rho=0.80$ ),

$$
\left[S_{\mathrm{SO}}-S_{\mathrm{EH}}\right] / N k=s_{0}(\text { squares })=5.497-\ln (4)-3.842=0.27 .
$$

The hard-cube entropy constant is somewhat less than that for hard spheres [17]. For cubes, with

$$
\Delta(P V / N k T) \equiv(P V / N k T)_{\mathrm{Cell}}-(P V / N k T)_{\mathrm{SO}},
$$

integration into the stable solid phase gives the entropy constant as follows:

$$
\begin{aligned}
s_{0}(\text { cubes }) & =(S / N k)_{\mathrm{SO}}-\left[(S / N k)_{\mathrm{FV}}+\ln (8)\right]=(S / N k)_{\mathrm{SO}}-(S / N k)_{\mathrm{EH}} \\
& =2.21-2.08=0.13 .
\end{aligned}
$$

Similarly, the last line of Table 2, corresponding to $\rho=0.70$, gives:

$$
\left[S_{\mathrm{SO}}-S_{\mathrm{EH}}\right] / N k=7.565-\ln (8)-5.346=0.13 \text {. }
$$


The hard-cube configurational integral near close packing exceeds that of the EyringHirschfelder cell theory by a factor of $e^{0.13}=1.14$. For hard spheres the corresponding factor is $e^{0.216}=1.24$. In the following section we consider the usefulness of these entropy estimates in locating phase equilibria for squares and cubes.

\section{Entropy and the Melting Transitions for Squares and Cubes}

Entropy plays a key role in establishing the nature of the melting transition for squares and cubes. At a fixed density the hard-particle phase having the greater entropy has also the lesser Helmholtz' free energy, $A=E-T S$, and is the stable phase. Thus the relative stability of the fluid and the solid is determined by their relative entropies.

The difference in entropy between the stable fluid and the less-stable single-occupancy solid was called the "communal entropy" by Kirkwood [23]. The communal entropy, absent in the single-occupancy solid, would be restored if multiple occupancy of all the cells were allowed. Notice that Tonks' exact calculation of the "hard-rod" partition function [21], mentioned in the Introduction, correctly accounts for multiple occupancy in the simplest one-dimensional case.

The communal entropy difference, fluid minus single-occupancy solid, is equal to $N k$ in the low density limit. The communal entropy gets smaller as the melting transition is approached, and finally vanishes at the density of the melting solid. In addition to this numberindependent effect there is an $N$-dependent contribution $\Delta S_{\text {com }}=k \ln N / N$ which can be ascribed to fluctuations [26]. As a result, the fluid gains in stability as $N$ increases, so that the melting transition tends to higher pressures and densities with increasing $N$.

Figures 5 and 6 show the communal entropy for squares and cubes based on trapezoidal rule integration of the fluid and single-occupancy solid data. The hard-square data match, nearly perfectly, the expected vanishing of the communal entropy (and equivalence of the Helmholtz free energies) at the phase transition density, 0.79. At that density both the entropies and the pressures of the two phases, fluid and single-occupancy solid, are nearly equal. Because the second-derivative isothermal bulk moduli,

$$
B_{T}=-V(\partial P / \partial V)_{T}=V\left(\partial^{2} A / \partial V^{2}\right)_{T},
$$

differ such a transition is called "second-order" rather than first. Of course numerical work cannot distinguish between such a second-order transition and a very weak first-order one,

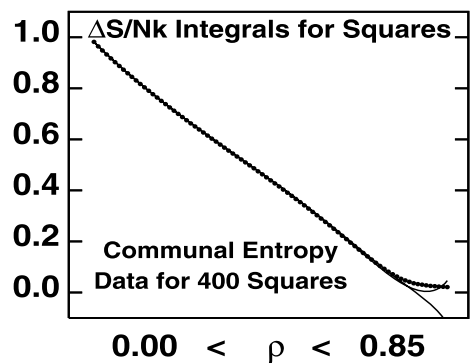

Fig. 5 Entropy differences calculated by integration of the dynamic data for 400 hard parallel squares. The points represent the "communal entropy", the difference between the fluid and single-occupancy solid entropies. The upper line, with a minimum at $\rho=0.80$ represents the entropy from the virial series through $B_{7}$. The lower line is based on the hard-square Padé approximant given in the Appendix. The 85 fluid and solid simulations used to construct the entropy differences used 400000 and 4000000 collisions, respectively 


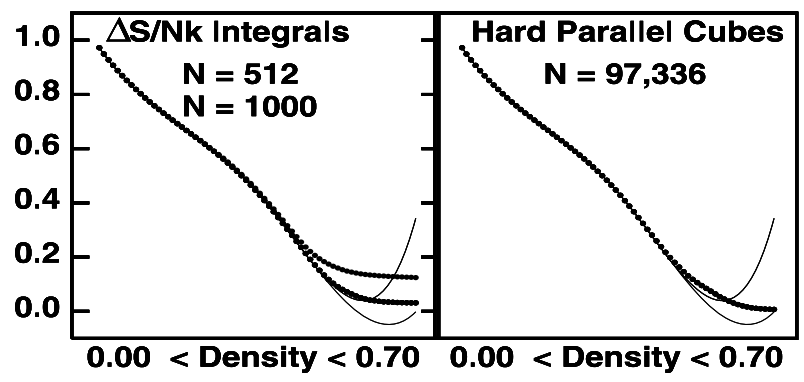

Fig. 6 Entropy differences calculated by integration of the hard-cube dynamic data. The points represent the difference between the fluid and solid entropies. The lines represents the predictions of the truncated virial series (above) and the Padé approximant given in the Appendix (below). The points for 512 and 1000 cubes were calculated from 70 simulations using $1000 N$ collisions. The 97,336-particle data, with nearly a billion collisions per point, are fully consistent with the single-occupancy simulations with an entropy difference of less than $0.01 \mathrm{~N} k$ at the maximum density shown here, $\rho=0.70$

Table 3 Compressibility factors for hard squares in the vicinity of the melting transition. 10,000 collisions per particle for $N=$ 100 and 400; 1000 collisions per particle for $N=900$ and 1600

\begin{tabular}{llllllll}
\hline$\rho$ & $Z_{100}$ & $Z_{400}$ & $Z_{900}$ & $Z_{1600}$ & $Z_{\text {virial }}$ & $Z_{\text {Padé }}$ & $Z_{\mathrm{FV}}$ \\
\hline 0.65 & 5.724 & 5.655 & 5.637 & 5.630 & 5.714 & 5.678 & 5.161 \\
0.70 & 6.549 & 6.682 & 6.641 & 6.608 & 6.724 & 6.740 & 6.122 \\
0.75 & 7.535 & 7.822 & 7.827 & 7.892 & 7.924 & 8.181 & 7.464 \\
0.80 & 9.410 & 9.510 & 9.488 & 9.484 & 9.472 & 10.426 & 9.472 \\
\hline
\end{tabular}

with slight differences in the densities of coexisting phases. The numerical work does make it clear that the difference between the solid and fluid densities, if any, is less than 0.01, considerably smaller than the corresponding solid-fluid density difference for hard disks $[10,25]$.

Figure 5 illustrates the variation of communal entropy with density for 400 hard squares, shown as points, together with the predictions of the truncated virial series through $B_{7}$ (line with a minimum at $\rho=0.80$ ) and those of the Padé approximant (the lower line). The number dependence seen in Table 3 can be avoided now by simulating systems of thousands of particles for millions of collisions. Such simulations are quite feasible on desktop computers.

For cubes the number dependence complicates an analysis. Systems with no more than 512 particles exhibit an irregular behavior in the region of confusion near the center of Fig. 4. The unconstrained data for 1000 cubes, shown in Fig. 6 and abstracted in Table 2, are not quite consistent with the single-occupancy calculations. The high-density entropy discrepancy is about $0.03 N k$.

We took advantage of the University of Manchester cluster of processors to complete an accurate unconstrained isotherm for $46 \times 46 \times 46=97,336$ hard cubes. More data from that machine will be forthcoming [27]. The corresponding entropy data are shown in Fig. 6. Figure 7 displays the difference between the 1000-cube and 97,336-cube compressibility factors.

The interpretation of the relatively-smooth data for squares is more straightforward. See Fig. 8. The Rees [6] reached the conclusion that squares have no first-order phase transition and the lack of difference between the "fluid" and "solid" equations of state near $\rho=0.79$ is quite consistent with this point of view. 
Fig. 7 Number-dependence of the pressure. 31 simulations with $1000 \mathrm{~N}$ collisions of 1000 cubes are compared with those with $10,000 N$ collisions of 97,336 cubes

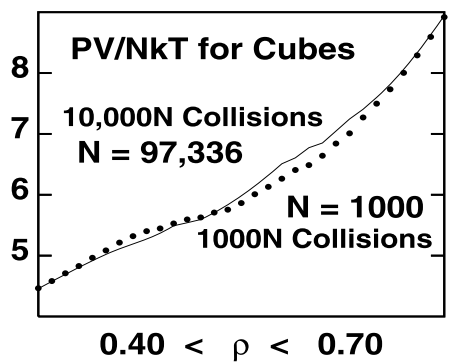

Fig. 8 Single-speed molecular dynamics pressure and integrated entropy for 400 fluid squares and 900 single-occupancy solid squares using a density interval of 0.01
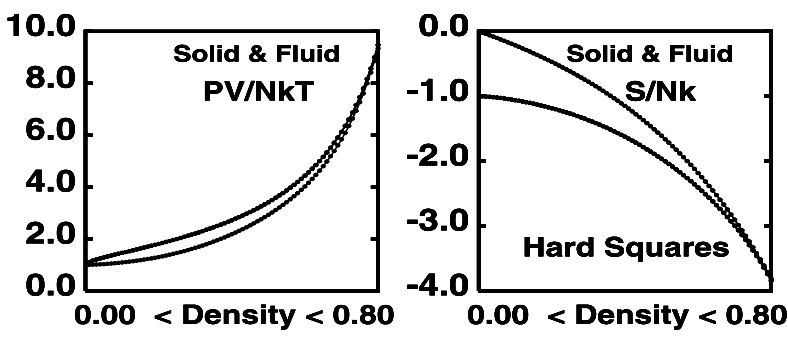

Fig. 9 Pressure data for 512 cubes in the region of confusion. Run lengths of $10 N, 100 N$, $1000 N$, and $10000 N$ collisions are indicated with four increasing dot sizes and a density interval of 0.01 . The curves are (from top to bottom) the Padé approximant, the seven-term virial series, and the free volume theory

Fig. 10 Pressure data for 1000 cubes in the region of confusion. Run lengths of $10 \mathrm{~N}, 100 \mathrm{~N}$, $1000 N$, and $10000 N$ collisions are indicated with four increasing dot sizes. The curves are (from top to bottom) the Padé approximant, the seven-term virial series, and the free volume theory
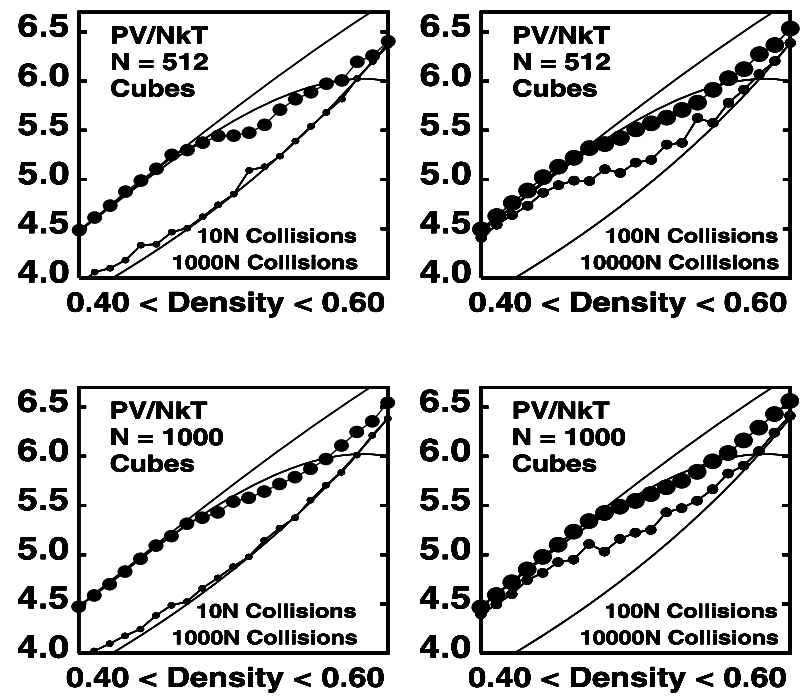

Cubes exhibit much more hysteresis and number dependence than do squares. Figures 9 and 10 show the relatively slow convergence of the pressure for densities in the region of confusion. $10 \mathrm{~N}$ collisions are scarcely enough to distinguish the pressure from the free volume theory. Longer runs, with $10^{4} \mathrm{~N}$ collisions, show that with increasing time the pressure gradually rises to a level between the truncated seven-term series and the somewhat higherpressure Padé approximant. We have included some longer-run data, for both squares and cubes, in Tables 3 and 4.

We also measured an "irregular" (fluid $\rightarrow$ glassy) isotherm for hard cubes. We placed $(N<11 \times 11 \times 11)$ particles randomly on a regular array of $11 \times 11 \times 11=1331$ lattice sites in a volume $V=1728$. Thus the initial state was a perfect lattice with many vacan- 
Table 4 Compressibility factors for unconstrained periodic hard cubes in the vicinity of the melting transition. 10,000 collisions per particle

\begin{tabular}{lllllllll}
\hline$\rho$ & $Z_{64}$ & $Z_{216}$ & $Z_{512}$ & $Z_{1000}$ & $Z_{1728}$ & $Z_{\text {virial }}$ & $Z_{\text {Padé }}$ & $Z_{\mathrm{FV}}$ \\
\hline 0.45 & 4.733 & 5.062 & 5.127 & 5.106 & 5.067 & 5.075 & 5.101 & 4.279 \\
0.50 & 4.887 & 5.377 & 5.509 & 5.547 & 5.599 & 5.594 & 5.713 & 4.847 \\
0.55 & 5.461 & 5.777 & 5.912 & 5.948 & 5.971 & 5.943 & 6.288 & 5.535 \\
0.60 & 6.264 & 6.386 & 6.534 & 6.563 & 6.602 & 6.000 & 6.815 & 6.387 \\
0.65 & 7.286 & 7.450 & 7.513 & 7.529 & 7.521 & 5.608 & 7.292 & 7.476 \\
0.70 & 8.650 & 8.868 & 8.913 & 8.920 & 8.921 & 4.565 & 7.725 & 8.921 \\
\hline
\end{tabular}

Fig. 11 Pressure data for $N=700,720,740, \ldots, 1200$ cubes in a volume 1728 . Each simulation includes $1000 \mathrm{~N}$ collisions. Initial positions were chosen randomly from an $11 \times 11 \times 11$ lattice fitting the volume. The results from the 26 simulations are joined by a heavy line. The dashed lines show the 1000-cube isotherm, the seven-term virial series, and the free-volume theory in the region of confusion

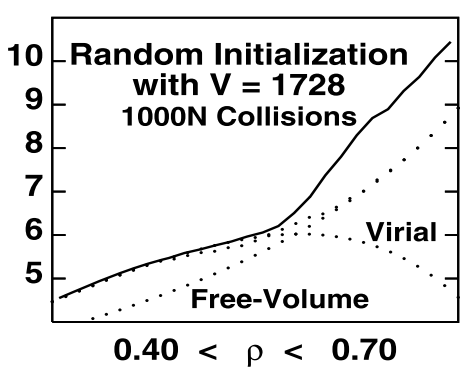

cies. Some of the resulting pressures are shown in Fig. 11, compared there with the 1000particle isotherm, the seven-term virial series, and the free-volume theory. It is evident that at densities of 0.57 and above the irregular isotherm deviates substantially from that of a "magic-number" system selected to "fit" the periodic boundaries perfectly.

The communal entropy for squares is relatively easy to compute. Even 400 squares are sufficient to give a smooth equation of state with a communal entropy close to zero at a density of 0.80 . See Fig. 5. To check this conclusion we have studied the hard-square density region $(0.60 \leq \rho \leq 0.80)$ carefully, with systems of $100,400,900$, and 1600 particles, using simulations of at least one million collisions. Some results are summarized in Table 3 and plotted in Fig. 8. The missing entropy in the integrated dynamic pressure is about an order of magnitude smaller for squares than for cubes, of the order $0.015 N k$ rather than $0.15 N k$. The calculated free energies for the fluid and solid phases merge very smoothly at a density of about 0.793 so that there is no sharp phase transition in the two-dimensional case. In order to make a reproducible estimate for the transition location we represent the hard-square fluid with the truncated virial series and the hard-square solid by the free-volume equation of state. The two pressures are equal at $\rho=0.793$ :

$$
P_{\text {virial }}(0.793)=P_{\mathrm{fv}}(0.793)=\rho k T Z=7.242 k T
$$

and the entropy difference agrees precisely with the Rees' estimate and the hard-disk value:

$$
\begin{aligned}
& {\left[S_{\text {virial }}(0.793)-S_{\text {ideal }}(0.793)\right] / N k=-3.765 ;} \\
& {\left[S_{\mathrm{fv}}(0.793)-S_{\text {ideal }}(0.793)\right] / N k=-5.424 \longrightarrow\left[S_{\text {virial }}(0.793)-S_{\mathrm{EH}}(0.793)\right] / N k=0.27_{3}}
\end{aligned}
$$

where the Eyring-Hirschfelder cell-model entropy exceeds that of the self-consistent freevolume theory for squares by $N k \ln 4=1.386 N k$. The definite change in slope required 
for consistency with the entropy data corresponds to a second-order phase change, with no volume difference between the two coexisting phases.

The detailed nature of the transition in the three-dimensional case awaits larger-scale simulations or additional diagnostics. The many curvature changes in the data shown here could easily mask one or more transitions of greater than second order.

\section{Summary and Conclusions}

Although computers are much faster now than in the pioneering days of Alder, Jacobsen, Wainwright, and Wood $[11,12]$ the hard-cube problem remains a challenge. Both the hardsquare and hard-cube phase transitions are weaker than the corresponding transitions for hard disks and spheres. The precise nature of the cube transition remains uncertain. The square transition appears to be second-order, with the pressure continuous and the compressibility discontinuous. The truncated virial series suggests a second-order transition while the higher-pressure Padé version of the fluid would correspond to a first-order transition a bit weaker than that found for hard disks. Such virial/Padé extrapolations of the pressure data are useful tools for analyzing the results for either squares or cubes.

For hard cubes in the solid phase, the free-volume equation of state and the ordinary cell model are excellent descriptions of both the pressure and the entropy (apart from an additive constant) for hard cubes, just as they were for squares. The characterization and appearance of the hard-cube solid phase could be sharpened by (1) an evaluation of the shear moduli, $C_{44}$ and $\left[C_{11}-C_{12}\right] / 2$ and (2) a study of the dependence of the diffusion coefficient on density. Both projects are research challenges. Protocols for measuring $C_{44}$ and $D$ in the solid phase require innovative boundary conditions.

Jagla [13] found a first-order melting transition for freely-rotating cubes. He also studied the parallel-cube model using constant-pressure simulations, and described a "continuous" melting transition at a density of $0.48 \pm 0.02$. Groh and Mulder presented an evenhanded criticism of Jagla's work [14], based on their own more extensive constant-pressure simulations. Groh and Mulder found a transition density of $0.53_{3} \pm 0.01$. In their view too the melting of hard parallel cubes is probably "continuous". The free-energy uncertainty in their work, $0.2 N k T$, exceeds ours by an order of magnitude. This difference seems quite large, in that single-occupancy simulations can easily achieve an accuracy an order of magnitude smaller, $0.01 N k T$. We are in agreement with these two assessments of hard-cube melting as "continuous".

Beyond this exploration of the melting transitions, this work has some interesting pedagogical consequences related to (i) the uncoupling of the configurational and kinetic parts of the partition function and (ii) the lack of coupling between the $x$ and $y$ and $z$ collisional momentum changes. The first of these uncouplings leads to successful but very simple implementations of quasiergodic single-speed dynamics. The second uncoupling makes the hard-parallel-cube gas an ideal mechanical thermometer, quite capable of measuring the independent tensor components of the kinetic temperature [28]. The simple linear trajectories of the present model can also be generalized to continuous potentials by using Lagrange multipliers to conserve energy along straightline trajectories:

$$
\ddot{x}=\Lambda \dot{x} ; \quad \ddot{y}=\Lambda \dot{y} ; \quad \ddot{z}=\Lambda \dot{z} .
$$

The Lyapunov instability of hard squares and cubes would also make an interesting topic for investigation. Although the collisions are between flat surfaces, without exponential 
growth in a scattering angle, at the same time it is clear that an offset in the particle coordinates will eventually (in a time roughly proportional to the offset) lead to a missed collision, with a totally different subsequent evolution. To relate these collisional bifurcations to standard Lyapunov analyses is another challenging research goal.

Acknowledgements We thank Les Woodcock, Francis Ree, and Leo Lue for encouragement, useful comments, and a selection of references. This work was presented at the University of Manchester in the spring of 2009.

Open Access This article is distributed under the terms of the Creative Commons Attribution Noncommercial License which permits any noncommercial use, distribution, and reproduction in any medium, provided the original author(s) and source are credited.

\section{Appendix}

Padé approximants to the seven-term virial series for squares and cubes can be obtained by equating the coefficients of like powers of the density. The symmetric approximants are

$$
P V / N k T=\frac{1-0.98155 \rho+0.32754 \rho^{2}-0.02760 \rho^{3}}{1-2.98155 \rho+3.29065 \rho^{2}-1.33090 \rho^{3}}
$$

for squares, and

$$
P V / N k T=\frac{1+1.45948 \rho+2.28842 \rho^{2}+0.91523 \rho^{3}}{1-2.54052 \rho+3.45049 \rho^{2}-1.35540 \rho^{3}}
$$

for cubes. Some details of the computation are given in Reference [17].

\section{References}

1. Zwanzig, R.W.: Virial coefficients of parallel square and parallel cube gases. J. Chem. Phys. 24, 855-856 (1956)

2. Hoover, W.G., De Rocco, A.G.: Sixth virial coefficients for gases of parallel hard lines, squares, and cubes. J. Chem. Phys. 34, 1059-1060 (1961)

3. Hoover, W.G., De Rocco, A.G.: Sixth and seventh virial coefficients for the parallel hard cube model. J. Chem. Phys. 36, 3141-3162 (1962)

4. Hoover, W.G., Alder, B.J.: Studies in molecular dynamics, IV. The pressure, collision rate, and their number dependence for hard disks. J. Chem. Phys. 46, 686-691 (1967)

5. Carlier, C., Frisch, H.L.: Molecular dynamics of hard parallel squares. Phys. Rev. A 6, 1153-1161 (1972)

6. Ree, F.H., Ree, T.: Statistical mechanics of the parallel hard squares in canonical ensemble. J. Chem. Phys. 56, 5434-5444 (1972)

7. Frisch, H.L., Roth, J., Krawchuk, B.D., Sofinski, P.: Molecular dynamics of nonergodic hard parallel squares with a Maxwellian velocity distribution. Phys. Rev. A 22, 740-744 (1980)

8. Lee, Y.S., Chae, D.G., Ree, T., Ree, F.H.: Computer simulations of a continuum system of molecules with a hard-core interaction in the grand canonical ensemble. J. Chem. Phys. 74, 6881-6887 (1981)

9. van Swol, F., Woodcock, L.V.: Percolation transition in the parallel hard cube model fluid. Mol. Simul. 1, 95-108 (1987)

10. Hoover, W.G., Ree, F.H.: Melting transition and communal entropy for hard spheres. J. Chem. Phys. 49, 3609-3617 (1968)

11. Wood, W.W., Jacobsen, J.D.: Preliminary results from a recalculation of the Monte Carlo equation of state of hard spheres. J. Chem. Phys. 27, 1207 (1957)

12. Alder, B.J., Wainwright, T.E.: Phase transition for a hard sphere system. J. Chem. Phys. 27, 1208 (1957)

13. Jagla, E.A.: Melting of hard cubes. Phys. Rev. E 58, 4701-4705 (1998) 
14. Groh, B., Mulder, B.: A closer look at crystallization of parallel hard cubes. J. Chem. Phys. 114, 36533658 (2001)

15. Mayer, J.E., Mayer, M.G.: Statistical Mechanics. Wiley, New York (1940)

16. Hoover, Wm.G.: Computational Statistical Mechanics. Elsevier, Amsterdam (1991). Available at the homepage http://williamhoover.info/book.pdf

17. Ree, F.H., Hoover, W.G.: Seventh virial coefficients for hard spheres and hard disks. J. Chem. Phys. 46, 4181-4197 (1967)

18. Clisby, N., McCoy, B.M.: Ninth and tenth order virial coefficients for hard spheres in D dimensions. J. Stat. Phys. 122, 15-57 (2005)

19. Hoover, W.G., Ashurst, W.T., Grover, R.: Exact dynamical basis for a fluctuating cell model. J. Chem. Phys. 57, 1259-1262 (1972)

20. Hoover, W.G., Hoover, N.E., Hanson, K.: Exact hard-disk free volumes. J. Chem. Phys. 70, 1837-1844 (1979)

21. Tonks, L.: The complete equation of state of one, two and three-dimensional gases of hard elastic spheres. Phys. Rev. 50, 955-963 (1936)

22. Hirschfelder, J.O., Curtiss, C.F., Bird, R.B.: The Molecular Theory of Gases and Liquids. Wiley, New York (1954). Chap. 4

23. Kirkwood, J.G.: Critique of the free volume theory of the liquid state. J. Chem. Phys. 18, 380-382 (1950)

24. Beyerlein, A.L., Rudd, W.G., Salsburg, Z.W., Buynoski, M.: Hard-square solids at high densities. J. Chem. Phys. 53, 1532-1540 (1970)

25. Hoover, W.G., Ree, F.H.: Use of computer experiments to locate the melting transition and calculate the entropy in the solid phase. J. Chem. Phys. 47, 4873-4878 (1967)

26. Hoover, W.G.: Entropy for small classical crystals. J. Chem. Phys. 49, 1981-1982 (1968)

27. Bannerman, M., Hoover, W.G., Hoover, C.G., Lue, L.: (in preparation)

28. Hoover, Wm.G., Hoover, C.G.: Nonlinear stresses and temperatures in transient adiabatic and shear flows via nonequilibrium molecular dynamics: three definitions of temperature. Phys. Rev. E 79, 046705 (2009) 